\title{
Effect of Foliar Nutrition on Economics of Soybean [Glycine max (L.) Merrill]
}

\author{
Indrapal Singh Paikra* and R. Lakpale \\ College of Agriculture, Raipur 492 012, Chhattisgarh, India \\ *E-mail: indrapaikra005@gmail.com
}

KEYWORDS Foliar Spray. Micronutrients. Quality. Soybean. Nutrient Uptake

\begin{abstract}
A field experiment was conducted during Kharif season of 2015 at the Research cum Instructional Farm, Indira Gandhi Krishi Vishwavidyalaya, Raipur to evaluate the effect of foliar nutrition on economics of soybean [Glycine max (L.) Merrill] under Vertisols of Chhattisgarh plains. The experiment was laid out in Randomized Block Design with three replications. The results revealed that application of RDF + spray of DAP @ 2 percent at pod initiations stages of crop growth resulted significantly higher uptake of nutrients $\mathrm{N}, \mathrm{P}, \mathrm{K}$, and also positive effect on micronutrients and economics compared to the application of RDF + Water spray at pod initiation stage and RDF.
\end{abstract}

\section{INTRODUCTION}

Soybean is a cheapest source of vegetable oil as well as protein. It contains about 40 percent protein, well balanced in essential amino acids, 20 percent oil rich with poly unsaturated fatty acids specially Omega 6 and Omega 3 fatty acids, 6-7 percent total mineral, 5-6 percent crude fiber and 17-19 percent carbohydrates. The protein quality of soybean is equivalent to that of meat, milk products and eggs. It is generally grown as a rainy season crop under rainfed situation. Thus, it is a "miracle bean" having many advantages. Soybean is recognized as golden bean because of its high nutritional values and economic importance. Soybean [Glycine max (L.) Merrill] is one of the most important oilseed crops in the world and it is also wonder crop of the twentieth century. The crop also helps in increasing the fertility level of soil through symbiotic nitrogen fixation. Foliar spraying is one alternative approach through micro nutrients are made to crop in liquid form through foliage (Nasiri et al. 2010). Foliar application of microelements is more beneficial than soil application. Since application rates are lesser as compared to soil application, same application could be obtained easily and crop reacts to nutrient application immediately (Zayed et al. 2011). Foliar spraying of microelements is very helpful when the roots cannot provide essential micro nutrients to the crop (Kinaci and Gulmezoglu 2007). Moreover, soil pollution would be a major problem by micronutrients soil application. As people are con- cerned about the environment, foliar sprays of nutrients are better than soil application. (Bozorgi et al. 2011).

Foliar application of macro and micro nutrients was more beneficial to legumes (Zayed et al. 2011). However adequate information on the effect of foliar application of Nitrogen, Phosphorus, Potassium, Molybdenum, Boron and Zinc on soybean was not available in Chhattisgarh agro-climatic condition. Considering above state facts, it has been proposed to study the effect of foliar nutrition of Nitrogen, Phosphorus, Potassium, Molybdenum, Boron and Zinc on growth and yield of soybean. Keeping the above fact in mind, the present investigation entitled Effect of Foliar Nutrition on uptake of Nutrients and Economics of Soybean [Glycine $\max ($ L.) Merrill]

\section{RESULTS AND DISCUSSION}

\section{Nutrient Uptake or Concentration (\%)}

It has been observed from Table 1 that foliar application of RDF+ spray of DAP @ 2\% at pod initiation Nutrient uptake or concentration (\%) of soybean recorded highest it might be due to the application of micronutrients in the form of foliar spray responded well and might be helpful to absorb other nutrients also in balance amount resulted more concentration of $\mathrm{N}, \mathrm{P}, \mathrm{K}, \mathrm{Mo}, \mathrm{B}$ and zinc in plant also reported by Mittra et al. (1987). 
Table 1: Nutrient uptake and concentration (\%) of soybean as affected by different treatments of micronutrients

\begin{tabular}{|c|c|c|c|c|c|c|}
\hline Treatments & $\begin{array}{c}\text { Nutrient } \\
N\left(k g h^{-1}\right) \\
\text { Stover }\end{array}$ & $\begin{array}{c}\text { Uptake } \\
\mathrm{P}_{2} \mathrm{O}_{5}\left(\mathrm{~kg} \mathrm{ha}^{-1}\right) \\
\text { Stover }\end{array}$ & $\begin{array}{c}\text { By } \mathrm{K}_{2} \mathrm{O} \\
\left(\mathrm{kg} \mathrm{ha}^{-1}\right) \\
\text { Stover }\end{array}$ & $\begin{array}{c}\text { Plants } \\
\mathrm{Mo}(\mathrm{Mg} \\
\left.\mathrm{kg}^{-1}\right)\end{array}$ & $\begin{array}{c}\text { Stover } \\
\text { B(Mg kg }{ }^{-1} \\
\text { Stover }\end{array}$ & $\begin{array}{c}\mathrm{Zn} \\
\left(\mathrm{Mg} \mathrm{kg}^{-1}\right) \\
\text { Stover }\end{array}$ \\
\hline $\mathrm{T}_{1}:$ RDF + Water spray at pod initiation & 184.6 & 26.00 & 113.8 & 0.02 & 0.02 & 0.02 \\
\hline $\mathrm{T}_{2}^{1}$ : RDF + Urea $2 \%$ spray at pod initiation & 185.8 & 28.00 & 115.6 & 0.12 & 0.02 & 0.03 \\
\hline $\mathrm{T}_{3}^{2}: \mathrm{RDF}+\mathrm{DAP} 2 \%$ spray at pod initiation & 190.1 & 30.00 & 117.2 & 0.04 & 0.03 & 0.04 \\
\hline $\mathrm{T}_{4}^{3}: \mathrm{RDF}+\mathrm{MOP} \quad 0.5 \%$ at pod initiation & 185.3 & 28.00 & 116.0 & 0.03 & 0.02 & 0.03 \\
\hline $\mathrm{T}_{5}^{4}: \mathrm{RDF}+19: 19: 19$ (NPK) $2 \%$ at pod initiatio & on 185.1 & 28.00 & 115.5 & 0.02 & 0.02 & 0.02 \\
\hline $\mathrm{T}_{6}^{5}: \mathrm{RDF}+$ Molybdenum $0.5 \%$ at pod initiation & 184.5 & 26.00 & 115.1 & 0.04 & 0.03 & 0.03 \\
\hline $\mathrm{T}_{7}^{6}:$ RDF + Boron $0.5 \%$ at pod initiation & 184.8 & 28.00 & 114.6 & 0.02 & 0.05 & 0.02 \\
\hline $\mathrm{T}_{8}^{\prime}: \mathrm{RDF}+$ Zinc Chelated $0.5 \%$ at pod initiatio & on 184.5 & 28.00 & 114.4 & 0.02 & 0.02 & 0.14 \\
\hline $\mathrm{T}_{9}^{8}: \mathrm{RDF}$ only & 184.2 & 26.00 & 113.5 & 0.02 & 0.02 & 0.02 \\
\hline S Em \pm & 1.548 & 0.354 & 0.522 & 0.032 & 0.011 & 0.041 \\
\hline $\mathrm{CD}(\mathrm{P}=0.05)$ & 4.641 & 1.062 & 1.565 & NS & NS & NS \\
\hline
\end{tabular}

\section{Balance Sheet for Available Micronutrients}

Foliar application of RDF+ spray of DAP @ 2 percent at pod initiation Nutrient uptake or concentration (\%) of soybean recorded highest (Tables 2, 3, 4). The maximum buildup of nutrients it might be due to through the high activity of root nodules which help the atmospheric nitrogen fixation which turn increases the nutrient status of the soil and also positive response to $\mathrm{P}, \mathrm{K}$, and micronutrients.

\section{Economics of Soybean}

Maximum gross return, net return and benefit: cost ratio was recorded under application of RDF + spray of DAP @ 2 percent in soybean (Table 5). The increase in gross and net return is obviously due to higher seed yield. Less input cost and higher economical yield might be resultant in increase the B: $\mathrm{C}$ ratio. Similar result was also reported by Kumar et al. (2015) spray of DAP @ 2 percent twice at flower initiation

Table 2: Balance sheet of available soil nitrogen as influenced by foliar spray of micronutrients

\begin{tabular}{|c|c|c|c|c|c|c|c|}
\hline Treatments & $\begin{array}{c}\text { Initial } \\
\text { soil } \\
\text { status } \\
\left(\mathrm{Kg} \mathrm{ha}^{-1}\right)\end{array}$ & $\begin{array}{l}\text { Nutrient } \\
\text { added } \\
\left(\mathrm{Kg} \mathrm{ha}^{-1}\right)\end{array}$ & $\begin{array}{c}\text { Total } \\
\text { Nutri- } \\
\text { ent } \\
\text { uptake } \\
\left(K^{\prime} h a^{-1}\right)\end{array}$ & $\begin{array}{l}\text { Expec- } \\
\quad \text { ted } \\
\text { nutrient } \\
\text { balance } \\
\left(\text { Kg ha }^{-1}\right)\end{array}$ & $\begin{array}{c}\text { Bala- } \\
\text { nce of } \\
\text { avail- } \\
\text { able K } \\
\text { after } \\
\text { harvest } \\
\text { applied } \\
\text { gain/loss }\end{array}$ & $\begin{array}{c}\text { Appa- } \\
\text { rent } \\
\text { gain/ } \\
\text { loss } \\
\left(K^{\prime} h a^{-1}\right)\end{array}$ & $\begin{array}{c}\text { Build } \\
\text { up (+) } \\
\text { orde- } \\
\text { pletion } \\
(-) \text { of } \\
\text { avail- } \\
\text { able } \\
K(\mathrm{~kg} \\
\left.\mathrm{ha^{-1 }}\right)\end{array}$ \\
\hline $\begin{array}{l}\mathrm{T}_{1}: \mathrm{RDF}+\text { Water spray } \\
\text { at pod initiation }\end{array}$ & 210 & 30 & 184.6 & 55.4 & 220 & 164.6 & 10 \\
\hline $\begin{array}{l}\mathrm{T}_{2}: \text { RDF + Urea } 2 \% \text { spray } \\
\text { at pod initiation }\end{array}$ & 210 & 30 & 185.8 & 54.2 & 219 & 164.9 & 9.1 \\
\hline $\begin{array}{l}\mathrm{T}_{3}: \mathrm{RDF}+\mathrm{DAP} 2 \% \text { spray } \\
\text { at pod initiation }\end{array}$ & 210 & 30 & 195.1 & 44.9 & 218 & 173.4 & 8.3 \\
\hline $\begin{array}{l}\mathrm{T}_{4}: \mathrm{RDF}+\mathrm{MOP} \quad 0.5 \% \text { at } \\
\text { pod initiation }\end{array}$ & 210 & 30 & 185.3 & 54.7 & 217 & 162.8 & 7.5 \\
\hline $\begin{array}{l}\mathrm{T}_{5}: \mathrm{RDF}+19: 19: 19(\mathrm{NPK}) \\
2 \% \text { at pod initiation }\end{array}$ & 210 & 30 & 185.1 & 54.9 & 215 & 162.2 & 7.1 \\
\hline $\begin{array}{l}\mathrm{T}_{6}: \mathrm{RDF}+\text { Molybdenum } \\
0.5 \% \text { at pod initiation }\end{array}$ & 210 & 30 & 184.5 & 55.5 & 216 & 159.8 & 5.3 \\
\hline $\begin{array}{l}\mathrm{T}_{7}: \mathrm{RDF}+\text { Boron } 0.5 \% \\
\text { at pod initiation }\end{array}$ & 210 & 30 & 184.8 & 55.2 & 215 & 160.1 & 5.3 \\
\hline $\begin{array}{c}\mathrm{T}_{8}: \mathrm{RDF}+\text { Zinc Chelated } \\
0.5 \% \text { at pod initiation }\end{array}$ & 210 & 30 & 184.5 & 55.5 & 215 & 159.5 & 5 \\
\hline $\mathrm{T}_{9}: \mathrm{RDF}$ only & 210 & 30 & 184.2 & 55.8 & 215 & 159.6 & 5.4 \\
\hline
\end{tabular}


Table 3: Balance sheet of available soil phosphorus as influenced by foliar spray of micronutrients

\begin{tabular}{|c|c|c|c|c|c|c|c|}
\hline Treatments & $\begin{array}{c}\text { Initial } \\
\text { soil } \\
\text { status } \\
\left(\mathrm{Kg} \mathrm{ha}^{-1}\right)\end{array}$ & $\begin{array}{l}\text { Nutrient } \\
\text { added } \\
\left(K g a^{-1}\right)\end{array}$ & $\begin{array}{c}\text { Total } \\
\text { Nutri- } \\
\text { ent } \\
\text { uptake } \\
\left(\mathrm{Kg} \mathrm{ha}^{-1}\right)\end{array}$ & $\begin{array}{c}\text { Expec- } \\
\text { ted } \\
\text { nutrient } \\
\text { balance } \\
\left(\text { Kg ha }^{-1}\right)\end{array}$ & $\begin{array}{c}\text { Bala- } \\
\text { nce of } \\
\text { avail- } \\
\text { able K } \\
\text { after } \\
\text { harvest } \\
\text { Applied } \\
\text { gain/loss }\end{array}$ & $\begin{array}{c}\text { Appa- } \\
\text { rent } \\
\text { gain/ } \\
\text { loss } \\
\left(K g h a^{-1}\right)\end{array}$ & $\begin{array}{c}\text { Build } \\
\text { up (+) } \\
\text { orde- } \\
\text { pletion } \\
(-) \text { of } \\
\text { avail- } \\
\text { able } \\
K(\mathrm{~kg} \\
\mathrm{ha^{-1 }}\end{array}$ \\
\hline $\begin{array}{l}\mathrm{T}_{1}: \mathrm{RDF}+\text { Water spray } \\
\text { at pod initiation }\end{array}$ & 16.4 & 60 & 26 & 50.4 & 40.3 & 10.0 & 23.9 \\
\hline $\begin{array}{l}\mathrm{T}_{2}: \mathrm{RDF}+\text { Urea } 2 \% \text { spray } \\
\text { at pod initiation }\end{array}$ & 16.4 & 60 & 28 & 48.4 & 42.7 & 5.7 & 26.3 \\
\hline $\begin{array}{l}\mathrm{T}_{3}: \mathrm{RDF}+\mathrm{DAP} 2 \% \text { spray } \\
\text { at pod initiation }\end{array}$ & 16.4 & 60 & 30 & 46.4 & 43.9 & 2.5 & 27.5 \\
\hline $\begin{array}{l}\mathrm{T}_{4}: \mathrm{RDF}+\mathrm{MOP} 0.5 \% \\
\text { at pod initiation }\end{array}$ & 16.4 & 60 & 28 & 48.4 & 42.6 & 5.8 & 26.2 \\
\hline $\begin{array}{l}\mathrm{T}_{5}: \mathrm{RDF}+19: 19: 19(\mathrm{NPK}) \\
2 \% \text { at pod initiation }\end{array}$ & 16.4 & 60 & 28 & 48.4 & 44.9 & 3.5 & 28.5 \\
\hline $\begin{array}{c}\mathrm{T}_{6}: \mathrm{RDF}+\text { Molybdenum } \\
0.5 \% \text { at pod initiation }\end{array}$ & 16.4 & 60 & 26 & 50.4 & 46.9 & 3.5 & 30.5 \\
\hline $\begin{array}{l}\mathrm{T}_{7}: \mathrm{RDF}+\text { Boron } 0.5 \% \text { at } \\
\text { pod initiation }\end{array}$ & 16.4 & 60 & 28 & 48.4 & 42.7 & 5.7 & 26.3 \\
\hline $\begin{array}{c}\mathrm{T}_{8}: \mathrm{RDF}+\text { Zinc Chelated } \\
0.5 \% \text { at pod initiation }\end{array}$ & 16.4 & 60 & 28 & 48.4 & 42.9 & 5.5 & 26.5 \\
\hline $\mathrm{T}_{9}:$ RDF only & 16.4 & 60 & 26 & 50.4 & 44.0 & 6.4 & 27.6 \\
\hline
\end{tabular}

Table 4: Balance sheet of available soil potash as influenced by foliar spray of micronutrients

\begin{tabular}{|c|c|c|c|c|c|c|c|}
\hline Treatments & $\begin{array}{c}\text { Initial } \\
\text { soil } \\
\text { status } \\
\left(\mathrm{Kg} \mathrm{ha}^{-1}\right)\end{array}$ & $\begin{array}{l}\text { Nutrient } \\
\text { added } \\
\left(\text { Kg ha }^{-1}\right)\end{array}$ & $\begin{array}{c}\text { Total } \\
\text { Nutri- } \\
\text { ent } \\
\text { uptake } \\
\left(K^{\prime} h^{-1}\right)\end{array}$ & $\begin{array}{l}\text { Expec- } \\
\quad \text { ted } \\
\text { nutrient } \\
\text { balance } \\
\left(\text { Kg ha }^{-1}\right)\end{array}$ & $\begin{array}{c}\text { Bala- } \\
\text { nce of } \\
\text { avail- } \\
\text { able K } \\
\text { after } \\
\text { harvest } \\
\text { Applied } \\
\text { gain/loss }\end{array}$ & $\begin{array}{c}\text { Appa- } \\
\text { rent } \\
\text { gain/ } \\
\text { loss } \\
\left(K g h a^{-1}\right)\end{array}$ & $\begin{array}{c}\text { Build } \\
\text { up (+) } \\
\text { orde- } \\
\text { pletion } \\
(-) \text { of } \\
\text { avail- } \\
\text { able } \\
K(\mathrm{~kg} \\
\left.\mathrm{ha^{-1 }}\right)\end{array}$ \\
\hline $\begin{array}{l}\mathrm{T}_{1}: \mathrm{RDF}+\text { Water spray } \\
\text { at pod initiation }\end{array}$ & 330 & 30 & 113.8 & 246.2 & 340.6 & 94.4 & 10.6 \\
\hline $\begin{array}{l}\mathrm{T}_{2}: \mathrm{RDF}+\text { Urea } 2 \% \text { spray } \\
\text { at pod initiation }\end{array}$ & 330 & 30 & 115.6 & 244.4 & 337.5 & 93.1 & 7.5 \\
\hline $\begin{array}{l}\mathrm{T}_{3}: \mathrm{RDF}+\mathrm{DAP} 2 \% \text { spray } \\
\text { at pod initiation }\end{array}$ & 330 & 30 & 117.2 & 242.3 & 336.1 & 93.8 & 6.1 \\
\hline $\begin{array}{l}\mathrm{T}_{4}: \mathrm{RDF}+\mathrm{MOP} \quad 0.5 \% \\
\text { at pod initiation }\end{array}$ & 330 & 30 & 116.0 & 244.0 & 338.0 & 94.8 & 8.8 \\
\hline $\begin{array}{l}\mathrm{T}_{5}: \mathrm{RDF}+19: 19: 19(\mathrm{NPK}) \\
2 \% \text { at pod initiation }\end{array}$ & 330 & 30 & 115.5 & 244.5 & 339.0 & 94.5 & 9.0 \\
\hline $\begin{array}{l}\mathrm{T}_{6}: \mathrm{RDF}+\text { Molybdenum } 0.5 \% \\
\text { at pod initiation }\end{array}$ & 330 & 30 & 115.1 & 244.9 & 337.0 & 92.6 & 7.5 \\
\hline $\begin{array}{l}\mathrm{T}_{7}: \mathrm{RDF}+\text { Boron } 0.5 \% \\
\text { at pod initiation }\end{array}$ & 330 & 30 & 114.4 & 245.6 & 338.0 & 93.0 & 8.6 \\
\hline $\begin{array}{c}\mathrm{T}_{8}: \mathrm{RDF}+\text { Zinc Chelated } \\
0.5 \% \text { at pod initiation }\end{array}$ & 330 & 30 & 114.0 & 245.6 & 339.0 & 93.9 & 9.5 \\
\hline $\mathrm{T}_{9}:$ RDF only & 330 & 30 & 113.5 & 246.5 & 336.0 & 90.0 & 6.5 \\
\hline
\end{tabular}

and pod formation stages of crop growth recorded higher gross returns (Rs. 36,500) and net returns (Rs. 20,090) followed by foliar spray of lo- cal variety at flower initiation and pod formation stages of crop growth with gross returns of Rs. 33,125 and net returns of Rs. 15,675. Water spray 
Table 5: Economics of soybean as affected by different treatments of micronutrients

\begin{tabular}{|c|c|c|c|c|}
\hline Treatments & $\begin{array}{c}\text { Cost of cultivation } \\
\left(R s h a^{-1}\right)\end{array}$ & $\begin{array}{l}\text { Gross return } \\
\quad\left(\text { Rs } h^{-1}\right)\end{array}$ & $\begin{array}{r}\text { Net return } \\
\quad\left(R s h a^{-1}\right)\end{array}$ & $B: C$ ratio \\
\hline $\mathrm{T}_{1}:$ RDF + Water spray at pod initiation & 19382 & 75445 & 56063 & 2.89 \\
\hline $\mathrm{T}_{2}^{1}:$ RDF + Urea $2 \%$ spray at pod initiation & 19452 & 77634 & 58182 & 2.99 \\
\hline $\mathrm{T}_{3}^{2}: \mathrm{RDF}+\mathrm{DAP} 2 \%$ spray at pod initiation & 19632 & 79911 & 60279 & 3.07 \\
\hline $\mathrm{T}_{4}^{3}: \mathrm{RDF}+\mathrm{MOP} \quad 0.5 \%$ at pod initiation & 19427 & 78071 & 58644 & 3.02 \\
\hline $\mathrm{T}_{5}^{4}: \mathrm{RDF}+19: 19: 19(\mathrm{NPK}) 2 \%$ at pod initiation & 21632 & 75570 & 53938 & 2.49 \\
\hline $\mathrm{T}_{6}^{5}$ : RDF +Molybdenum $0.5 \%$ at pod initiation & 34382 & 67335 & 32953 & 0.96 \\
\hline $\mathrm{T}_{7}^{6}: \mathrm{RDF}+$ Boron $0.5 \%$ at pod initiation & 20922 & 72945 & 52023 & 2.49 \\
\hline $\mathrm{T}_{8}$ : RDF+ Zinc Chelated $0.5 \%$ at pod initiation & 21227 & 73655 & 52428 & 2.47 \\
\hline $\mathrm{T}_{9}^{\circ}:$ RDF only & 19182 & 71497 & 52315 & 2.73 \\
\hline SEm \pm & - & 2006 & 2006 & 0.098 \\
\hline CD $(P=0.05)$ & - & NS & NS & NS \\
\hline
\end{tabular}

recorded the least gross returns (Rs. 20,250) and net returns (Rs. 4,000), and the B: C ratio (2.22) was higher under the treatments where DAP @ 2 percent was applied twice at flower initiation and pod formation stages of crop growth.

\section{CONCLUSION}

Foliar spray of DAP @ 2 percent higher uptake of nutrients by plants and positive effect on yield and also registered significantly higher net return of (60279 Rs) with B: C ratio of 3.07. When compared with the application of RDF only. So farmer may adopt Foliar spray of DAP @ 2 percent for achieving higher yield and profit of their field.

\section{REFERENCES}

Anonymous 2015. Directorate of Economics and Statistics, Department of Agriculture and Cooperation. From <www.indiastat.com. $>$

Bozorgi HA, Azarpour E, Moradi M 2011. Effects of bio, mineral nitrogen fertilization and foliar zinc spraying on yield and yield components of faba bean. World Appl Sci J, 13(6): 1409-1414.

Ganapathy M, Baradhan G, Ramesh N 2008. Effect of foliar nutrition on reproductive efficiency and grain yield of rice fallow pulses. Legume Res, 31: 142144.
Kumar S, Basavarajappa R, Salakinkop SR, Hebbar M, Basavarajappa MP, Patil H Y 2013. Influence of foliar nutrition on performance of blackgram (Vigna mungo L.), nutrient uptake and economics under dry land ecosystems. Legume Research, 36(5).

Kumar CV, Vaiyapuri K, Amanullah MM, Gopalaswamy $\mathrm{G}$ 2013. Influence of foliar spray of nutrients on yield and economics of soybean (Glycine max L. Merill). Journal of Biological science, 13(6); 563565.

Kinaci E, Gulmezoglu N 2007. Grain yield and yield components of triticale upon application of different foliar fertilizers. Interciencia, 32(9): 624-628.

Mitra R, Pawar SE, Bhatia CR. 1987. Effect of Foliar Spray of Nutrients and Plant Growth Regulators (PGRs) for Yield Maximization in Blackgram. International Symposium, Bangkok, Thailand 16-20. AVRDC (Tropical Vegetable Information Service). Nasiri Y, Zehtab-Salmasi S, Nasrullahzadeh S, Najafi N, Ghassemi-Golezani K 2010. Effects of foliar application of micronutrients (Fe and $\mathrm{Zn}$ ) on flower yield and essential oil of chamomile (Matricaria chamomilla L.). Journal of Medicinal Plants Research, 4(17): 1733- 1737.

Yakadri M, Ramesh T 2002. Effect of soil application of potassium and DAP spray in blackgram (Vigna mungo L.). Madras Agric J, 89: 147-149.

Zayed A, Salem M, Sharkawy M 2011. Effect of different micronutrient treatments on rice (Oriza sativa L.) growth and yield under saline soil conditions. World J Agric Sci, 7(2): 179-184.

Paper received for publicaiton on November 2016 Paper accepted for publication on December 2017 